\title{
On a Number of Colors in Cyclically Interval Edge Colorings of Simple Cycles
}

\author{
Rafayel R. Kamalian \\ Institute for Informatics and Automation Problems, The National Academy of Sciences of Republic of Armenia, \\ Yerevan, Republic of Armenia \\ Email: rrkamalian@yahoo.com
}

Received November 23, 2012; revised December 23, 2012; accepted December 31, 2012

\begin{abstract}
A proper edge $t$-coloring of a graph $G$ is a coloring of its edges with colors $1,2, \cdots, t$ such that all colors are used, and no two adjacent edges receive the same color. A cyclically interval $t$-coloring of a graph $G$ is a proper edge $t$-coloring of $G$ such that for each its vertex $x$, either the set of colors used on edges incident to $x$ or the set of colors not used on edges incident to $x$ forms an interval of integers. For an arbitrary simple cycle, all possible values of $t$ are found, for which the graph has a cyclically interval $t$-coloring.
\end{abstract}

Keywords: Proper Edge Coloring; Cyclically Interval Coloring; Simple Cycle

\section{Introduction}

We consider undirected, simple, finite and connected graphs. For a graph $G$, we denote by $V(G)$ and $E(G)$ the sets of its vertices and edges, respectively. The set of edges of $G$ incident with a vertex $x \in V(G)$ is denoted by $J_{G}(x)$. For any $x \in V(G)$, $d_{G}(x)$ denotes the degree of the vertex $x$ in $G$. For a graph $G, \Delta(G)$ denotes the maximum degree of a vertex of $G$. A simple cycle with $n$ edges $(n \geq 3)$ is denoted by $C(n)$. A simple path with $n$ edges $(n \geq 1)$ is denoted by $P(n)$. The terms and concepts that we do not define can be found in [1].

For an arbitrary finite set $A$, we denote by $|A|$ the number of elements of $A$. The set of positive integers is denoted by $\mathbb{N}$. For any subset $D$ of the set $\mathbb{N}$, we denote by $D_{(0)}$ and $D_{(1)}$ the subsets of all even and all odd elements of $D$, respectively.

An arbitrary nonempty subset of consecutive integers is called an interval. An interval with the minimum element $p$ and the maximum element $q$ is denoted by $[p, q]$. An interval $D$ is called a $h$-interval if $|D|=h$.

For any real number $\xi$, we denote by $\lfloor\xi\rfloor(\lceil\xi\rceil)$ the maximum (minimum) integer which is less (greater) than or equal to $\xi$.

For any positive integer $k$ define

$$
\varepsilon(k) \equiv 1+\left\lfloor\frac{k}{2}\right\rfloor-\left\lceil\frac{k}{2}\right\rceil .
$$

For any nonnegative integer $k$ define

$$
\delta(k) \equiv \begin{cases}0, & \text { if } k=0 \\ 1 & \text { otherwise }\end{cases}
$$

A function $\varphi: E(G) \rightarrow[1, t]$ is called a proper edge $t$-coloring of a graph $G$, if all colors are used, and no two adjacent edges receive the same color.

The minimum value of $t$ for which there exists a proper edge $t$-coloring of a graph $G$ is denoted by $\chi^{\prime}(G)[2]$.

If $G$ is a graph, and $\varphi$ is its proper edge $t$-coloring, where $t \in\left[\chi^{\prime}(G),|E(G)|\right]$, then we define

$$
U(G, \varphi) \equiv\{e \in E(G) / 1<\varphi(e)<t\} .
$$

If $E_{0} \subseteq E(G), \quad t \in\left[\chi^{\prime}(G),|E(G)|\right]$, and $\varphi$ is a proper edge $t$-coloring of a graph $G$, then we set

$$
\varphi\left[E_{0}\right] \equiv\left\{\varphi(e) / e \in E_{0}\right\} .
$$

A proper edge $t$-coloring $\left(t \in\left[\chi^{\prime}(G),|E(G)|\right]\right) \varphi$ of a graph $G$ is called an interval $t$-coloring of $G$ [35] if for any $x \in V(G)$, the set $\varphi\left[J_{G}(x)\right]$ is a

$d_{G}(x)$-interval. For any $t \in \mathbb{N}$, we denote by $\mathfrak{N}_{t}$ the set of graphs for which there exists an interval $t$-coloring. Let

$$
\mathfrak{N}=\bigcup_{t \geq 1} \mathfrak{N}_{t} .
$$

For any $G \in \mathfrak{N}$, we denote by $w_{\text {int }}(G)$ and $W_{\text {int }}(G)$ the minimum and the maximum possible value of $t$, respectively, for which $G \in \mathfrak{N}_{t}$. For a graph $G$, let us 
set $\theta(G) \equiv\left\{t \in \mathbb{N} / G \in \mathfrak{N}_{t}\right\}$.

A proper edge $t$-coloring $\left(t \in\left[\chi^{\prime}(G),|E(G)|\right]\right) \varphi$ of a graph $G$ is called a cyclically interval $t$-coloring of $G$, if for any $x \in V(G)$, at least one of the following two conditions holds:

1) $\varphi\left[J_{G}(x)\right]$ is a $d_{G}(x)$-interval,

2) $[1, t] \backslash \varphi\left[J_{G}(x)\right]$ is a $\left(t-d_{G}(x)\right)$-interval.

For any $t \in \mathbb{N}$, we denote by $\mathfrak{M}_{t}$ the set of graphs for which there exists a cyclically interval $t$-coloring. Let

$$
\mathfrak{M} \equiv \bigcup_{t \geq 1} \mathfrak{M}_{t}
$$

For any $G \in \mathfrak{M}$, we denote by $w_{c y c}(G)$ and $W_{c y c}(G)$ the minimum and the maximum possible value of $t$, respectively, for which $G \in \mathfrak{M}_{t}$. For a graph $G$, let us set $\Theta(G) \equiv\left\{t \in \mathbb{N} / G \in \mathfrak{M}_{t}\right\}$.

It is clear that for any $G \in \mathfrak{N}$, an arbitrary interval $t$ coloring $(t \in \theta(G))$ of a graph $G$ is also a cyclically interval $t$-coloring of $G$. Thus, for any $t \in \mathbb{N}$, $\mathfrak{N}_{t} \subseteq \mathfrak{M}_{t}$ and $\mathfrak{N} \subseteq \mathfrak{M}$. Let us also note that for an arbitrary graph $G, \theta(G) \subseteq \Theta(G)$. It is also clear that for any $G \in \mathfrak{N}$, the following inequality is true:

$$
\Delta(G) \leq \chi^{\prime}(G) \leq w_{c y c}(G) \leq w_{\text {int }}(G)
$$

and

$$
W_{\text {int }}(G) \leq W_{\text {cyc }}(G) \leq|E(G)| .
$$

In $[5,6]$, for any tree $G$, it is proved that $G \in \mathfrak{N}$, $\theta(G)$ is an interval, and the exact values of the parameters $w_{\text {int }}(G), W_{\text {int }}(G)$ are found. In [7,8], for any tree $G$, it is proved that $\Theta(G)=\theta(G)$. Some interesting results on cyclically interval $t$-colorings and related topics were obtained in [9-14].

In this paper, for any integer $n \geq 3$, it is proved that $C(n) \in \mathfrak{M}$, and the set $\Theta(C(n))$ is found.

\section{Main Results}

Remark 1. Clearly, for any integer $n \geq 3$,

$$
\chi^{\prime}(C(n))=3-\varepsilon(n),|E(C(n))|=n .
$$

Therefore, if $t \notin[3-\varepsilon(n), n]$, then a proper edge $t$ coloring of $C(n)$ does not exist, and $C(n) \notin \mathfrak{N}_{t}$.

Remark 2. It is not difficult to see that for any integer $k \geq 2, C(2 k) \in \mathfrak{N}$ and $\theta(C(2 k))=[2, k+1]$.

Proposition 1. For any integer $n \geq 3, C(n) \in \mathfrak{M}$, $n \in \Theta(C(n)) . \Theta(C(3))=\{3\}, \Theta(C(4))=\{2,3,4\}$.

Proof is trivial.

Theorem 1. For any integers $n$ and $t$, satisfying the conditions $n \geq 5$ and $t \in[3-\varepsilon(n), n], C(n) \notin \mathfrak{M}_{t}$ if and only if

$$
t \in\left[4+\varepsilon(n) \cdot\left(\frac{n}{2}+\varepsilon\left(\left\lfloor\frac{n}{2}\right\rfloor\right)-2\right), n-1\right]_{(\varepsilon(n))} .
$$

Proof. First let us prove, that if $n \in \mathbb{N}, n \geq 5$ and

$$
t \in\left[4+\varepsilon(n) \cdot\left(\frac{n}{2}+\varepsilon\left(\left\lfloor\frac{n}{2}\right\rfloor\right)-2\right), n-1\right]_{(\varepsilon(n))}
$$

then $C(n) \notin \mathfrak{M}_{t}$.

Assume the contrary: there are $n_{0} \in \mathbb{N}, n_{0} \geq 5$ and

$$
t_{0} \in\left[4+\varepsilon\left(n_{0}\right) \cdot\left(\frac{n_{0}}{2}+\varepsilon\left(\left\lfloor\frac{n_{0}}{2}\right\rfloor\right)-2\right), n_{0}-1\right]_{\left(\varepsilon\left(n_{0}\right)\right)},
$$

for which a cyclically interval $t_{0}$-coloring $\alpha$ of the graph $C\left(n_{0}\right)$ exists.

Let us construct a graph $H_{00}$ removing from the graph $C\left(n_{0}\right)$ the subset $U\left(C\left(n_{0}\right), \alpha\right)$ of its edges. Let us construct a graph $H_{0}$ removing from the graph $H_{00}$ all its isolated vertices.

Case $A . H_{0}$ is a connected graph.

Let us denote by $F$ the simple path with pendant edges $e^{\prime}$ and $e^{\prime \prime}$ which is isomorphic to the graph $P\left(n_{0}-\left|E\left(H_{0}\right)\right|+2\right)$.

Case A.1. $n_{0}$ is odd.

Clearly, $t_{0} \in\left[4, n_{0}-1\right]_{(0)}$. It means that $t_{0}$ is an even number, satisfying the inequality $4 \leq t_{0} \leq n_{0}-1$.

Case A.1.1. $\left|E\left(H_{0}\right)\right|$ is odd.

Clearly, $\left|E\left(H_{0}\right)\right| \geq 3$. Since $\alpha$ is a cyclically interval $t_{0}$-coloring of $C\left(n_{0}\right)$, we conclude from the definition of $H_{0}$, that for a graph $F$, there exists an interval $\left(t_{0}-1\right)$-coloring $\beta_{1}$ with $\beta_{1}\left(e^{\prime}\right)=\beta_{1}\left(e^{\prime \prime}\right)$. Consequently, the number $n_{0}-\left|E\left(H_{0}\right)\right|+2$ is odd, what contradicts the same parity of $n_{0}$ and $\left|E\left(H_{0}\right)\right|$.

Case A.1.2. $\left|E\left(H_{0}\right)\right|$ is even.

Clearly, $\left|E\left(H_{0}\right)\right| \geq 2$. Since $\alpha$ is a cyclically interval $t_{0}$-coloring of $C\left(n_{0}\right)$, we conclude from the definition of $H_{0}$, that for a graph $F$, there exists an interval $t_{0}$-coloring $\beta_{2}$ with $\beta_{2}\left(e^{\prime}\right)=1$ and $\beta_{2}\left(e^{\prime \prime}\right)=t_{0}$. Consequently, the number $n_{0}-\left|E\left(H_{0}\right)\right|+2$ is even, what contradicts the different parity of $n_{0}$ and $\left|E\left(H_{0}\right)\right|$.

Case A.2. $n_{0}$ is even.

Clearly, $t_{0} \in\left[\frac{n_{0}}{2}+2+\varepsilon\left(\frac{n_{0}}{2}\right), n_{0}-1\right]_{(1)}$. It means that $t_{0}$ is an odd number, satisfying the inequality

$\frac{n_{0}}{2}+2+\varepsilon\left(\frac{n_{0}}{2}\right) \leq t_{0} \leq n_{0}-1$.

Case A.2.1. $\left|E\left(H_{0}\right)\right|$ is odd.

Clearly, $\left|E\left(H_{0}\right)\right| \geq 3$. Since $\alpha$ is a cyclically interval $t_{0}$-coloring of $C\left(n_{0}\right)$, we can conclude from the 
definition of $H_{0}$, that for a graph $F$, there exists an interval $\left(t_{0}-1\right)$-coloring $\beta_{3}$ with $\beta_{3}\left(e^{\prime}\right)=\beta_{3}\left(e^{\prime \prime}\right)$. Consequently,

$$
\begin{aligned}
n_{0} & >n_{0}-\left|E\left(H_{0}\right)\right|+2=|E(F)| \\
& \geq 2 t_{0}-3 \geq n_{0}+1+2 \cdot \varepsilon\left(\frac{n_{0}}{2}\right)>n_{0},
\end{aligned}
$$

which is impossible.

Case A.2.2. $\left|E\left(H_{0}\right)\right|$ is even.

Clearly, $\left|E\left(H_{0}\right)\right| \geq 2$. Since $\alpha$ is a cyclically interval $t_{0}$-coloring of $C\left(n_{0}\right)$, we can conclude from the definition of $H_{0}$, that for a graph $F$, there exists an interval $t_{0}$-coloring $\beta_{4}$ with $\beta_{4}\left(e^{\prime}\right)=1$ and $\beta_{4}\left(e^{\prime \prime}\right)=t_{0}$. Since $t_{0}$ is odd, the number $n_{0}-\left|E\left(H_{0}\right)\right|+2$ is also odd, but it is impossible because of the same parity of $n_{0}$ and $\left|E\left(H_{0}\right)\right|$.

Case $B . H_{0}$ is a graph with $m$ connected components, $m \geq 2$.

Assume that:

1) $H_{1}, \cdots, H_{m}$ are connected components of $H_{0}$ numbered in succession at bypassing of the graph $C\left(n_{0}\right)$ in some fixed direction,

2) $v_{1}, \cdots, v_{n_{0}}$ are vertices of $C\left(n_{0}\right)$ numbered in succession at bypassing mentioned in 1$)$,

3) $e_{1}, \cdots, e_{n_{0}}$ are edges of $C\left(n_{0}\right)$ numbered in succession at bypassing mentioned in 1$)$,

4) $v_{1} \in V\left(H_{1}\right), \quad v_{2} \in V\left(H_{1}\right), \quad v_{n_{0}} \notin V\left(H_{1}\right)$, $e_{1}=\left(v_{1}, v_{2}\right)$.

Define functions

$$
\begin{aligned}
& \zeta:[1, m] \rightarrow\left[1, n_{0}-1\right], \\
& \eta:[1, m] \rightarrow\left[1, n_{0}-1\right], \\
& y:[1,2 m] \rightarrow\{0,1\}
\end{aligned}
$$

as follows. For any $i \in[1, m]$, set:

$$
\begin{aligned}
& \zeta(i) \equiv \min \left\{k / e_{k} \in E\left(H_{i}\right)\right\}, \\
& \eta(i) \equiv \max \left\{k / e_{k} \in E\left(H_{i}\right)\right\} .
\end{aligned}
$$

For any $j \in[1,2 m]$, set

$$
y(j) \equiv \begin{cases}\delta\left(\alpha\left(e_{\zeta\left(\frac{j+1}{2}\right)}\right)-1\right), & \text { if } j \text { is odd } \\ \delta\left(\alpha\left(e_{\eta\left(\frac{j}{2}\right)}\right)-1\right), & \text { if } j \text { is even. }\end{cases}
$$

Now let us define subgraphs $H_{1}^{\prime}, \cdots, H_{m}^{\prime}$ of the graph $C\left(n_{0}\right)$.

For any $i \in[1, m-1]$, let $H_{i}^{\prime}$ be the subgraph of $C\left(n_{0}\right)$ induced by the subset

$$
\left\{v_{\eta(i)}, v_{\eta(i)+1}, \cdots, v_{\zeta(i+1)}, v_{\zeta(i+1)+1}\right\}
$$

of its vertices. Let $H_{m}^{\prime}$ be the subgraph of $C\left(n_{0}\right)$ induced by the subset

$$
\left\{v_{\eta(m)}, v_{\eta(m)+1}, \cdots, v_{n_{0}}, v_{1}, v_{2}\right\}
$$

of its vertices.

Let

$$
\begin{gathered}
M_{1} \equiv\left\{i \in[1, m] / 1 \in \alpha\left[E\left(H_{i}^{\prime}\right)\right]\right\}, \\
M_{2} \equiv\left\{i \in[1, m] / t_{0} \in \alpha\left[E\left(H_{i}^{\prime}\right)\right]\right\} .
\end{gathered}
$$

For any $j \in[1,2 m]$, we define a point $\pi_{j}$ of the 2dimensional rectangle coordinate system by the following way: $\pi_{j} \equiv(j, y(j))$.

Let us define a graph $\tilde{H}$. Set

$$
\begin{aligned}
& V(\tilde{H}) \equiv\left\{\pi_{1}, \cdots, \pi_{2 m}\right\}, \\
& E(\tilde{H}) \equiv\left\{\left(\pi_{2 m}, \pi_{1}\right)\right\} \cup\left\{\left(\pi_{j}, \pi_{j+1}\right) / j \in[1,2 m-1]\right\} .
\end{aligned}
$$

Clearly, $\tilde{H} \cong C(2 m)$.

Let

$$
\begin{aligned}
& E_{1}(\tilde{H}) \equiv\left\{\left(\pi_{2 q-1}, \pi_{2 q}\right) / q \in[1, m]\right\}, \\
& E_{2}(\tilde{H}) \equiv E(\tilde{H}) \backslash E_{1}(\tilde{H}) .
\end{aligned}
$$

An edge $\left(\pi^{\prime}, \pi^{\prime \prime}\right)$ of the graph $\tilde{H}$ is called horizontal if the points $\pi^{\prime}$ and $\pi^{\prime \prime}$ have the same ordinate.

Let us denote by $E_{-}(\tilde{H})$ the set of all horizontal edges of the graph $\tilde{H}$. Set $E_{\mid}(\tilde{H}) \equiv E(\tilde{H}) \backslash E_{-}(\tilde{H})$. It is easy to note that the numbers $\left|E_{-}(\tilde{H})\right|$ and $\left|E_{\mid}(\tilde{H})\right|$ are both even.

Now let us define a function $\psi: E(\tilde{H}) \rightarrow\left[1, n_{0}-1\right]$ by the following way. For an arbitrary $e \in E(\tilde{H})$ set:

$$
\begin{aligned}
& \psi(e) \\
& \equiv\left\{\begin{array}{l}
\left|E\left(H_{q}\right)\right|, \text { if } e=\left(\pi_{2 q-1}, \pi_{2 q}\right), \text { where } q \in[1, m] \\
\left|E\left(H_{q}^{\prime}\right)\right|, \text { if } e=\left(\pi_{2 q}, \pi_{2 q+1}\right), \text { where } q \in[1, m-1] \\
\left|E\left(H_{m}^{\prime}\right)\right|, \text { if } e=\left(\pi_{2 m}, \pi_{1}\right) .
\end{array}\right.
\end{aligned}
$$

Clearly,

$$
\sum_{e \in E(\tilde{H})} \psi(e)=n_{0}+2 m .
$$

Case B.1. $n_{0}$ is odd.

Clearly, $t_{0} \in\left[4, n_{0}-1\right]_{(0)}$. It means that $t_{0}$ is an even number, satisfying the inequality $4 \leq t_{0} \leq n_{0}-1$. It is not difficult to see that in this case, for an arbitrary $e \in E_{-}(\tilde{H}), \psi(e)$ is odd, and, moreover, for an arbitrary $e \in E_{\mid}(\tilde{H}), \psi(e)$ is even. Since $\left|E_{-}(\tilde{H})\right|$ is 
even, we conclude that the odd number

$$
n_{0}+2 m=\sum_{e \in E_{-}(\tilde{H})} \psi(e)+\sum_{e \in E_{(}(\tilde{H})} \psi(e)
$$

is represented as a sum of two even numbers, which is impossible.

Case B.2. $n_{0}$ is even.

Clearly,

$$
t_{0} \in\left[\frac{n_{0}}{2}+2+\varepsilon\left(\frac{n_{0}}{2}\right), n_{0}-1\right]_{(1)} .
$$

It means that $t_{0}$ is an odd number, satisfying the inequality

$$
\frac{n_{0}}{2}+2+\varepsilon\left(\frac{n_{0}}{2}\right) \leq t_{0} \leq n_{0}-1
$$

It is not difficult to see that in this case, for an arbitrary $e \in E_{2}(\tilde{H}) \cup E_{-}(\tilde{H}), \psi(e)$ is odd, and, moreover, for an arbitrary $e \in E_{1}(\tilde{H}) \cap E_{\mid}(\tilde{H}), \psi(e)$ is even.

Case B.2.1. $\left|E_{2}(\tilde{H}) \cap E_{\mid}(\tilde{H})\right| \geq 2$.

In this case, evidently, there are different integers $i^{\prime}$ and $i^{\prime \prime}$ in the set $[1, m]$, for which there exist interval $t_{0}$-colorings $\beta^{\prime}$ and $\beta^{\prime \prime}$ of the graphs $H_{i^{\prime}}^{\prime}$ and $H_{i^{\prime \prime}}^{\prime}$, respectively. Consequently,

$$
\begin{aligned}
n_{0} & =\left|E\left(C\left(n_{0}\right)\right)\right| \geq\left|E\left(H_{i^{\prime}}^{\prime}\right) \cup E\left(H_{i^{\prime \prime}}^{\prime}\right)\right| \\
& =\left|E\left(H_{i^{\prime}}^{\prime}\right)\right|+\left|E\left(H_{i^{\prime \prime}}^{\prime}\right)\right|-\left|E\left(H_{i^{\prime}}^{\prime}\right) \cap E\left(H_{i^{\prime \prime}}^{\prime}\right)\right| \\
& \geq\left|E\left(H_{i^{\prime}}^{\prime}\right)\right|+\left|E\left(H_{i^{\prime \prime}}^{\prime}\right)\right|-2 \geq 2 t_{0}-2 \\
& \geq n_{0}+2+2 \varepsilon\left(\frac{n_{0}}{2}\right)>n_{0},
\end{aligned}
$$

which is impossible.

Case B.2.2. $\left|E_{2}(\tilde{H}) \cap E_{\mid}(\tilde{H})\right|=1$.

Without loss of generality assume that

$$
E_{2}(\tilde{H}) \cap E_{\mid}(\tilde{H})=\left\{e^{0}\right\} .
$$

Since $\left|E_{-}(\tilde{H})\right|$ is even, we conclude that the even number

$$
\begin{aligned}
& n_{0}+2 m \\
& =\sum_{e \in E_{-}(\tilde{H})} \psi(e)+\sum_{e \in E_{1}(\tilde{H})} \psi(e) \\
& =\sum_{e \in E_{2}(\tilde{H}) \cap E_{1}(\tilde{H})} \psi(e)+\sum_{e \in E_{1}(\tilde{H}) \cap_{1}(\tilde{H})} \psi(e)+\sum_{e \in E_{-}(\tilde{H})} \psi(e) \\
& =\psi\left(e^{0}\right)+\sum_{e \in E_{1}(\tilde{H}) \cap E_{1}(\tilde{H})} \psi(e)+\sum_{e \in E_{-}(\tilde{H})} \psi(e)
\end{aligned}
$$

is represented as a sum of one odd and two even numbers, which is impossible.

Case B.2.3. $\left|E_{2}(\tilde{H}) \cap E_{\mid}(\tilde{H})\right|=0$.
Clearly, for any $i \in[1, m]$, the set $\alpha\left[E\left(H_{i}^{\prime}\right)\right]$ contains exactly one of the colors 1 and $t_{0}$.

Case B.2.3.a). $M_{1} \neq \varnothing, M_{2}=\varnothing$.

It is not difficult to see that in this case there is $i_{1} \in M_{1}$, for which the set $\alpha\left[E\left(H_{i_{1}}^{\prime}\right)\right]$ contains the color $t_{0}-1$. It means that there exists an interval $\left(t_{0}-1\right)$-coloring of the graph $H_{i_{1}}^{\prime}$ which colors pendant edges of $H_{i_{1}}^{\prime}$ by the color 1. Consequently,

$$
n_{0}>\left|E\left(H_{i_{1}}^{\prime}\right)\right| \geq 2 t_{0}-3 \geq n_{0}+1+2 \varepsilon\left(\frac{n_{0}}{2}\right)>n_{0},
$$

which is impossible.

Case B.2.3.b). $M_{1}=\varnothing, M_{2} \neq \varnothing$.

It is not difficult to see that in this case there is $i_{2} \in M_{2}$, for which the set $\alpha\left[E\left(H_{i_{2}}^{\prime}\right)\right]$ contains the color 2 . It means that there exists an interval $\left(t_{0}-1\right)$ coloring of the graph $H_{i_{2}}^{\prime}$ which colors pendant edges of $H_{i_{2}}^{\prime}$ by the color 1. Consequently,

$$
n_{0}>\left|E\left(H_{i_{2}}^{\prime}\right)\right| \geq 2 t_{0}-3 \geq n_{0}+1+2 \varepsilon\left(\frac{n_{0}}{2}\right)>n_{0},
$$

which is impossible.

Case B.2.3.c). $M_{1} \neq \varnothing, M_{2} \neq \varnothing$.

Let us choose $i_{3} \in M_{1}$ and $i_{4} \in M_{2}$ satisfying the conditions

$$
\begin{aligned}
& \left|\alpha\left[E\left(H_{i_{3}}^{\prime}\right)\right]\right|=\max _{i \in M_{1}} \mid \alpha\left[E\left(H_{i}^{\prime}\right)\right], \\
& \left|\alpha\left[E\left(H_{i_{4}}^{\prime}\right)\right]\right|=\max _{i \in M_{2}} \mid \alpha\left[E\left(H_{i}^{\prime}\right)\right] .
\end{aligned}
$$

Let $j^{(3)}$ be the maximum color of the set

$$
\alpha\left[E\left(H_{i_{3}}^{\prime}\right)\right] .
$$

Let $j^{(4)}$ be the minimum color of the set

$$
\alpha\left[E\left(H_{i_{4}}^{\prime}\right)\right] .
$$

Clearly, $j^{(3)} \geq j^{(4)}-1$.

It is not difficult to see that there exists an interval $j^{(3)}$-coloring of the graph $H_{i_{3}}^{\prime}$ which colors pendant edges of $H_{i_{3}}^{\prime}$ by the color 1 . Hence,

$$
\left|E\left(H_{i_{3}}^{\prime}\right)\right| \geq 2 j^{(3)}-1 .
$$

It is not difficult to see that there exists an interval $\left(t_{0}-j^{(4)}+1\right)$-coloring of the graph $H_{i_{4}}^{\prime}$ which colors pendant edges of $H_{i_{4}}^{\prime}$ by the color 1. Hence,

$$
\left|E\left(H_{i_{4}}^{\prime}\right)\right| \geq 2 \cdot\left(t_{0}-j^{(4)}+1\right)-1=2 t_{0}-2 j^{(4)}+1 .
$$

Consequently, we obtain that

$$
\begin{aligned}
& n_{0}>\left|E\left(H_{i_{3}}^{\prime}\right) \cup E\left(H_{i_{4}}^{\prime}\right)\right|=\left|E\left(H_{i_{3}}^{\prime}\right)\right|+\left|E\left(H_{i_{4}}^{\prime}\right)\right| \\
& \geq 2 t_{0}+2\left(j^{(3)}-j^{(4)}\right) \geq 2 t_{0}-2 \geq n_{0}+2+2 \varepsilon\left(\frac{n_{0}}{2}\right)>n_{0},
\end{aligned}
$$


which is impossible.

Thus, we have proved that if $n \in \mathbb{N}, n \geq 5$ and

$$
t \in\left[4+\varepsilon(n) \cdot\left(\frac{n}{2}+\varepsilon\left(\left\lfloor\frac{n}{2}\right\rfloor\right)-2\right), n-1\right]_{(\varepsilon(n))},
$$

then $C(n) \notin \mathfrak{M}_{t}$.

Now let us prove that if

$$
n \in \mathbb{N}, \quad n \geq 5, \quad t \in[3-\varepsilon(n), n], C(n) \notin \mathfrak{M}_{t},
$$

then

$$
t \in\left[4+\varepsilon(n) \cdot\left(\frac{n}{2}+\varepsilon\left(\left\lfloor\frac{n}{2}\right\rfloor\right)-2\right), n-1\right]_{(\varepsilon(n))} .
$$

Assume the contrary. It means that there are $n_{0} \in \mathbb{N}$, $n_{0} \geq 5$, and $t_{0} \in\left[3-\varepsilon\left(n_{0}\right), n_{0}\right]$, which satisfy the conditions $C\left(n_{0}\right) \notin \mathfrak{M}_{t_{0}}$ and

$$
t_{0} \notin\left[4+\varepsilon\left(n_{0}\right) \cdot\left(\frac{n_{0}}{2}+\varepsilon\left(\left\lfloor\frac{n_{0}}{2}\right\rfloor\right)-2\right), n_{0}-1\right]_{\left(\varepsilon\left(n_{0}\right)\right)} .
$$

Case 1. $n_{0}$ is odd.

In this case $t_{0} \in\left[3, n_{0}\right]$ and $t_{0} \notin\left[4, n_{0}-1\right]_{(0)}$, and, therefore, $t_{0} \in\left[3, n_{0}\right]_{(1)}$. It means that there exists $m_{0} \in \mathbb{N}$, for which

$$
2 \leq m_{0}=\frac{t_{0}+1}{2} \leq \frac{n_{0}+1}{2} .
$$

Let us note that the equality $m_{0}=\frac{n_{0}+1}{2}$ implies $t_{0}=n_{0}$, which is incompatible with the condition $C\left(n_{0}\right) \notin \mathfrak{M}_{t_{0}}$. Hence, $n_{0}-2 m_{0} \geq 1$.

Now, to see a contradiction, it is enough to note that the existence of an interval $t_{0}$-coloring of a graph $P\left(2 m_{0}-1\right)$ with the existence of an interval 2-coloring of a graph $P\left(n_{0}-2 m_{0}+1\right)$ provides the existence of a cyclically interval $t_{0}$-coloring of the graph $C\left(n_{0}\right)$.

Case 2. $n_{0}$ is even.

In this case $t_{0} \in\left[2, n_{0}\right]$ and

$$
t_{0} \notin\left[\frac{n_{0}}{2}+2+\varepsilon\left(\frac{n_{0}}{2}\right), n_{0}-1\right]_{(1)},
$$

and, therefore,

$$
t_{0} \in\left[2, \frac{n_{0}}{2}+1\right] \cup\left(\left[\frac{n_{0}}{2}+3-\varepsilon\left(\frac{n_{0}}{2}\right), n_{0}\right]_{(0)}\right) .
$$

It follows from Remark 2 that

$$
t_{0} \in\left[\frac{n_{0}}{2}+3-\varepsilon\left(\frac{n_{0}}{2}\right), n_{0}\right]_{(0)} .
$$

Clearly, there exists $m_{0} \in \mathbb{N}$,

$$
m_{0} \leq \frac{1}{2}\left(\frac{n_{0}}{2}+\varepsilon\left(\frac{n_{0}}{2}\right)-1\right),
$$

for which

$$
t_{0}=\frac{n_{0}}{2}+1-\varepsilon\left(\frac{n_{0}}{2}\right)+2 m_{0} .
$$

Let us note that the equality

$$
m_{0}=\frac{1}{2}\left(\frac{n_{0}}{2}+\varepsilon\left(\frac{n_{0}}{2}\right)-1\right)
$$

implies $t_{0}=n_{0}$, which is incompatible with the condition $C\left(n_{0}\right) \notin \mathfrak{M}_{t_{0}}$. Hence,

$$
\frac{n_{0}}{2}+\varepsilon\left(\frac{n_{0}}{2}\right)-1-2 m_{0}
$$

is an even number, satisfying the inequality

$$
\frac{n_{0}}{2}+\varepsilon\left(\frac{n_{0}}{2}\right)-1-2 m_{0} \geq 2 \text {. }
$$

Now, to see a contradiction, it is enough to note that the existence of an interval $t_{0}$-coloring of a graph

$$
P\left(\frac{n_{0}}{2}+1-\varepsilon\left(\frac{n_{0}}{2}\right)+2 m_{0}\right)
$$

with the existence of an interval 2-coloring of a graph

$$
P\left(\frac{n_{0}}{2}+\varepsilon\left(\frac{n_{0}}{2}\right)-1-2 m_{0}\right)
$$

provides the existence of a cyclically interval $t_{0}$-coloring of the graph $C\left(n_{0}\right)$.

Thus, we have proved, that if $n \in \mathbb{N}, n \geq 5$,

$$
t \in[3-\varepsilon(n), n], C(n) \notin \mathfrak{M}_{t},
$$

then

$$
t \in\left[4+\varepsilon(n) \cdot\left(\frac{n}{2}+\varepsilon\left(\left\lfloor\frac{n}{2}\right\rfloor\right)-2\right), n-1\right]_{(\varepsilon(n))} .
$$

Theorem 1 is proved.

It means that we also have

Theorem 2. For an arbitrary integer $n \geq 5$,

$$
\begin{aligned}
& \Theta(C(n)) \\
& = \begin{cases}{[3, n]_{(1)},} & \text { if } n \text { is odd } \\
{\left[2, \frac{n}{2}+1\right] \cup\left(\left[\frac{n}{2}+3-\varepsilon\left(\frac{n}{2}\right), n\right]_{(0)}\right),} & \text { if } n \text { is even. }\end{cases}
\end{aligned}
$$

\section{Acknowledgements}

The author thanks P.A. Petrosyan and N.A. Khachatryan for their attention to this work. 


\section{REFERENCES}

[1] D. B. West, "Introduction to Graph Theory," PrenticeHall, Upper Saddle River, 1996.

[2] V. G. Vizing, "The Chromatic Index of a Multigraph," Kibernetika, Vol. 3, 1965, pp. 29-39.

[3] A. S. Asratian and R. R. Kamalian, "Interval Colorings of Edges of a Multigraph," Applied Mathematics, Vol. 5, Yerevan State University, 1987, pp. 25-34. (in Russian)

[4] A. S. Asratian and R. R. Kamalian, "Investigation of Interval Edge-Colorings of Graphs," Journal of Combinatorial Theory, Series B, Vol. 62, No. 1, 1994, pp. 34-43. doi:10.1006/jctb.1994.1053

[5] R. R. Kamalian, "Interval Edge Colorings of Graphs," Doctoral Dissertation, The Institute of Mathematics of the Siberian Branch of the Academy of Sciences of USSR, Novosibirsk, 1990. (in Russian)

[6] R. R. Kamalian, "Interval Colorings of Complete Bipartite Graphs and Trees," Preprint of the Computing Centre of the Academy of Sciences of Armenia, 1989. (in Russian)

[7] R. R. Kamalian, "On a Number of Colors in Cyclically Interval Edge Colorings of Trees," Research Report LiTHMAT-R-2010/09-SE, Linkoping University, 2010.

[8] R. R. Kamalian, “On Cyclically-Interval Edge Colorings of Trees," Buletinul Academiei de Stiinte a Republicii Moldova Matematica, Vol. 68, No. 1, 2012, pp. 50-58.

[9] A. Kotzig, "1-Factorizations of Cartesian Products of Regular Graphs," Journal of Graph Theory, Vol. 3, No. 1, 1979, pp. 23-34. doi:10.1002/jgt.3190030104

[10] J. J. Bartholdi, J. B. Orlin and H. D. Ratliff, "Cyclic Scheduling via Integer Programs with Circular Ones," Operations Research, Vol. 28, No. 5, 1980, pp. 1074 1085. doi:10.1287/opre.28.5.1074

[11] W. Dauscha, H. D. Modrow and A. Neumann, "On Cyclic Sequence Type for Constructing Cyclic Schedules," Zeitschrift für Operations Research, Vol. 29, No. 1, 1985, pp. 1-30.

[12] D. de Werra, N. V. R. Mahadev and P. Solot, "Periodic Compact Scheduling," ORWP 89/18, Ecole Polytechnique Fédérale de Lausanne, 1989.

[13] D. de Werra and Ph. Solot, "Compact Cylindrical Chromatic Scheduling," ORWP 89/10, Ecole Polytechnique Fédérale de Lausanne, 1989.

[14] R. R. Kamalian, "On Cyclically Continuous Edge Colorings of Simple Cycles," Proceedings of the Computer Science and Information Technologies Conference, Yerevan, 24-28 September 2007, pp. 79-80. (in Russian) 